\title{
Investigating the quasi-oscillatory behavior of electrical parameters with the concentration of D-glucose in aqueous solution
}

\author{
Subhadip Chakraborty ${ }^{1}$, Chirantan Das ${ }^{1}$, Rajib Saha ${ }^{1}$, Avishek Das ${ }^{1}$, Nirmal Kumar Bera ${ }^{2}$, Dipankar \\ Chattopadhyay $^{2}$, Anupam Karmakar ${ }^{1}$, Dhrubajyoti Chattopadhyay ${ }^{3}$, Sanatan Chattopadhyay ${ }^{1,4}$ \\ 1. Department of Electronic Science, University of Calcutta, 92 A.P.C. Road, Kolkata- 700009, India \\ 2. Department of Polymer Science and Technology, University of Calcutta, 92 A.P.C. Road, Kolkata- 700009, India \\ 3. Department of Biochemistry, University of Calcutta, 35, Ballygunge Circular Road, Kolkata - 700 019, India \\ 4. E-mail and correspondence to: scelc@caluniv.ac.in
}

\begin{abstract}
The impedance, capacitance and conductance of deionized waterglucose polar solution is measured by employing impedance spectroscopy and a quasi-oscillatory nature of variation with glucose content in the solution is observed. Such quasi-oscillatory nature is attributed to the randomly distributed water-water, waterglucose and glucose-glucose dipole interactions at the molecular level in the solution. A relevant analytical model is developed on the basis of such random distribution of the molecular dipoles and the experimental data agree well with those obtained from the theoretical model. The electrical parameters are measured in the frequency range of $100 \mathrm{~Hz}$ to $4 \mathrm{MHz}$ for the volume fractions of glucose with respect to water in the range of 0.1 to 0.5 . The impedance, capacitance and conductance are obtained to be in the range of $1.03 \mathrm{k} \Omega-112 \mathrm{k} \Omega, 34.9 \mathrm{pF}-1.66 \mathrm{nF}$, and $8.95 \mu \mathrm{S}-52.9$ $\mu \mathrm{S}$ respectively for the glucose volume fraction range considered.
\end{abstract}

Keywords: Quasi-oscillatory nature, DI water-glucose, polar solution, multi-body dipole correlation, dipole polarization, impedance spectroscopy.

\section{Introduction}

The electrical characterization of glucose in different solutions has been a topic of enormous research interest for several decades and to date research is being carried out around the globe to explore salient properties of these liquids due to the fundamental role of glucose as a fuel molecule in many biological processes [1-3]. Among various approaches, the electrical analysis of glucose solutions by impedance spectroscopy is becoming a very potential method [4-8].

Glucose is a monosaccharide with pyranose skeleton and also it is polar in nature due to the arrangement of its constituent hydrogen and oxygen atoms, which leads to an uneven distribution of electrons around the entire molecule [9]. Consequently, glucose in different base solutions exhibits very interesting electrical characteristics for its particular structure and polarization effect. Such electrical properties depend on the polarity of base solvents and the compositional concentration of glucose in the solution [1011]. As an example, a linear dependency of electrical parameters on the glucose content of its solution is observed $[3,12-13]$ in a conductive type buffer saline, however, such variation is different in a pure polar type solution like DI water. The water molecule is non-linear and the centers of its positive and negative charges do not coincide, thereby, making it a polar molecule and therefore the aqueous solution of glucose shows different electrical properties compared to an ionic solution.

Different theoretical models have been proposed to demonstrate the electrical behavior of glucose molecules in a solution. The Clausius-Mosotti equation explained the effective dielectric behavior of solutions containing nonpolar solutes [14-17]; however, it is not sufficient to analyze the similar behavior for solutions with polar solutes. Debye then developed a theory on the effective dielectric constant of a solution with polar molecules [15]. Onsager [16] and then Kirkwood [17] founded their models on the basis of Debye theory with the concept of continuum approach by considering the molecule to be surrounded by a continuous medium.

There are three types of dipole interactions present in the system of an aqueous solution of glucose: the water-water, water-glucose and glucose-glucose interactions [18-19]. Therefore, the net polarization must be a function of the compositional concentration of glucose in the system. When an external electric field is applied to the system, the orientations of the molecular dipoles inside it play a pivotal role on the net polarization of the system [20]. In most of the theoretical models, the multi-body dipole correlations are not clearly explained and the orientations of dipoles relevant to the constituent molecules in the system are not properly emphasized. Thus, the relationship between the effective dielectric constant with the variation of compositional concentration of glucose in a pure polar solution such as deionized (DI) water has not yet been adequately addressed. Some experimental results employing impedance spectroscopic techniques on DI water-glucose solutions are also available that indicate a non-linear quasi-oscillatory behavior of the dielectric constant and conductance of the solution with compositional variation of glucose $[4,21]$. However, no comprehensive theoretical model to describe such oscillatory behavior is available and therefore to develop a thorough understanding on the nature of dielectric behavior of a polar-polar solution a more generalized straightforward model is needed, including the dipole-dipole interactions, dipole orientations along with the applied electric field and also with each other. 
In this work, a theoretical model is developed for describing the quasi-oscillatory nature of dielectric constant of pure DI water-glucose mixture with the compositional variation of glucose content in the solution. Emphasis has been placed on the randomness of dipoles inside the bulk solution, which is in thermal equilibrium, since such statistical approach indeed is more generalized as this considers the dipole-dipole interactions of individual molecules and also their random orientations in the system. The analytical model has been verified with the help of experimental results of impedance, capacitance and conductance measured by employing the impedance spectroscopy of the DI water-glucose solution with varying glucose content.

\section{Materials and methods}

\section{Sample preparation}

Glucose stock solutions were prepared using electronic grade deionized (DI) water (Millipore ${ }^{\mathrm{TM}}$ ) and D-glucose powder (Merck) for thirteen different concentrations of glucose ranging from $15.4 \mathrm{gm} / \mathrm{dl}$ to $77.0 \mathrm{gm} / \mathrm{dl}$. Density of glucose is $1.54 \mathrm{gm} / \mathrm{cc}$ and hence equivalent volume fractions of glucose with respect to DI water in the solution range from 0.1 to 0.5 .

\section{Theoretical modeling of the DI water-glucose solution}

A bulk aqueous solution of glucose and DI water has been considered in the current work, where both DI water and glucose are polar in nature and hence their solution is also polar. To describe the dielectric behavior of such a polar medium, the solution is considered to be constituted of polar molecules with randomly oriented permanent dipoles of moment, $\vec{p}$. The schematic representation of such a polar solution is shown in Fig. 1(a). Since such individual dipoles are randomly oriented the net dipole moment of the system is zero, in the absence of any external applied electric field. However, when an electric field is applied then torques are generated on the individual dipoles to align themselves with the field, thereby developing a net dipole moment per unit volume or more precisely, giving rise to the polarization $\mathbf{P}$.

The potential energy of a polar molecule of dipole moment $\vec{p}$ and applied electric field $\vec{E}$ can be expressed as [23]

$$
W=-\vec{p} \cdot \vec{E}=-p E \cos \theta
$$

where $\theta$ is the angle between the vectors relevant to dipole moment and applied electric field.

Fig.1 (b) shows the solid angle that a dipole may form with the applied electric field. Now, since the system is in thermal equilibrium, the probability of any molecule having potential energy, $W$, due to its orientation to the electric field, is proportional to $e^{-W / k T}$, where $k$ is the Boltzmann's constant and $T$ is absolute temperature of the solution. Thus, the system follows Boltzmann statistics, and therefore, the number of dipoles per unit volume per unit solid angle for an angle $\theta$ with the direction of electric field, can be given as [24]

$$
n(\theta) \propto e^{-W / k T}
$$

Hence,

$$
n(\theta)=C e^{p E \cos \theta / k T}
$$

where $C$ is the proportionality constant. The solid angle (Fig. 1(b)) between $\theta$ and $(\theta+d \theta)$ is equal to $2 \pi \sin \theta d \theta$.

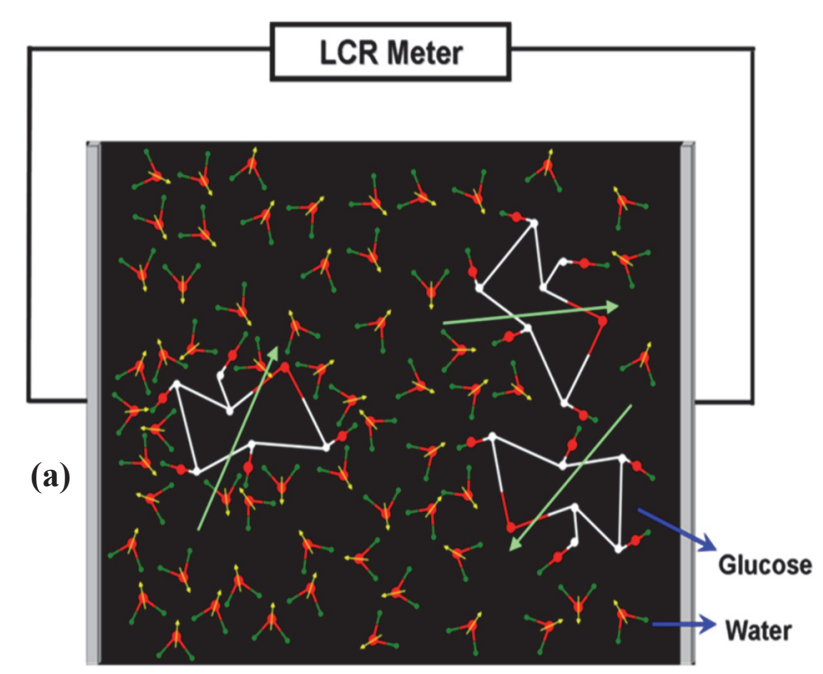

(b)

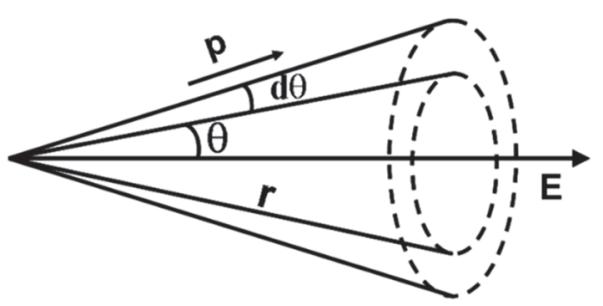

Fig. 1: (a) Schematic representation of water-water, glucose-water and glucose-glucose dipole interactions in glucose-DI water solution with randomly oriented dipoles under the effect of an external electric field; (b) Schematic diagram to describe the conical solid angle included between $\theta$ and $(\theta+d \theta)$.

Thus, the number of dipoles per unit volume lying in the conical solid angle of Fig. 1(b) is

$$
d n=C e^{\frac{p E \cos \theta}{k T}} 2 \pi \sin \theta d \theta
$$

Hence, the total number of molecules for all possible values of $\theta$ is equal to

$$
\int d n=n=\int C e^{p E \cos \theta / k T} 2 \pi \sin \theta d \theta
$$

where, 


$$
C=\frac{n}{2 \pi \int_{0}^{2 \pi} e^{p E \cos \theta / k T} 2 \pi \sin \theta d \theta}
$$

The value of $n$ has been calculated to be in the order of $10^{28} / \mathrm{m}^{3}$ considering the number of moles present in the homogeneous bulk solution. Now, the projection of $\vec{p}$ on the direction of electric field $\vec{E}$ is $p \cos \theta$ and the polarization, $\vec{P}=n \vec{p}$. Thus, the net contribution from the dipoles to polarization is

$$
\vec{P}=\int_{0}^{\pi} n(\theta) d \theta p \cos \theta
$$

Substituting the value of $C$ we obtain

$$
\vec{P}=n p \frac{\int_{0}^{\pi} e^{p E \cos \theta / k T} \sin \theta \cos \theta d \theta}{\int_{0}^{\pi} e^{p E \cos \theta / k T} \sin \theta d \theta}
$$

Further simplification of this expression gives

$$
\vec{P}=\frac{n p^{2}}{3 k T} \vec{E}
$$

Now, the electric displacement vector is given by, $\vec{D}=\varepsilon \vec{E}$ and according to Gauss's theorem for a dielectrics, there exists a certain amount of polarization charge in the system along with its total free charge [22] and further yields the electric displacement vector as $\vec{D}=\varepsilon_{0} \vec{E}+\vec{P}$; where $\varepsilon_{0}$ is the permittivity of free space. This leads to a relationship between the polarization and electric field

$$
\vec{P}=\left(\varepsilon-\varepsilon_{0}\right) \vec{E}
$$

Comparing for $\vec{P}$, we can write

$$
\begin{aligned}
\frac{n p^{2}}{3 k T} \vec{E} & =\left(\varepsilon-\varepsilon_{0}\right) \vec{E} \\
\frac{\varepsilon}{\varepsilon_{0}} & =\frac{n p^{2}}{3 k T \varepsilon_{0}}+1
\end{aligned}
$$

The solution of DI water and glucose will have three different types of interactions at the molecular level: the water-water, glucose-glucose and water-glucose dipoles that finally determine the overall dielectric behavior of the solution [25-26]. Therefore, the net polarization of an elementary volume, contributed from these three different components, can be written as

$$
\begin{aligned}
& p^{2}=\left(p_{W} \cos \theta_{W}\right)^{2}+\left(p_{G} \cos \theta_{G}\right)^{2} \\
& +2 p_{W} p_{G} \cos \theta_{W} \cos \theta_{G} \cos \varphi
\end{aligned}
$$

and the total dipole moment for the entire volume of the solution is

$$
\begin{aligned}
& \sum_{i} p_{i}^{2}=\sum_{i=1}^{N_{w}}\left(p_{w i} \cos \theta_{w i}\right)^{2}+\sum_{i=1}^{N_{G}}\left(p_{G i} \cos \theta_{G i}\right)^{2} \\
& +2 \sum_{i=1}^{N_{S}} p_{w i} p_{G i} \cos \theta_{w i} \cos \theta_{G i} \cos \phi_{i}
\end{aligned}
$$

where $p_{G}$ and $p_{W}$ correspond to the dipole moments of glucose and water, respectively, $\theta_{W}$ and $\theta_{G}$ represent the angles between the dipoles and applied field for water and glucose, respectively, and $\phi$ is the angle between the resultant dipole moments for water and glucose molecules in the system. $N_{W}, N_{G}$ and $N_{S}$ are the numbers of elementary volumes relevant to water, glucose and entire solution within the measuring cell, respectively. Thus, $N_{G} / N_{W}$ is equivalent to the volume fraction of glucose with respect to DI water in the solution.

Thus, the effective net dielectric constant of the system can be expressed as

$$
\begin{aligned}
& \varepsilon_{e f f}=\frac{\varepsilon}{\varepsilon_{0}}=\frac{n}{3 k T \varepsilon_{0}}\left[\sum_{i=1}^{N_{w}}\left(p_{w i} \cos \theta_{w i}\right)^{2}+\sum_{i=1}^{N_{G}}\left(p_{G i} \cos \theta_{G i}\right)^{2}\right. \\
& \left.+2 \sum_{i=1}^{N_{S}} p_{w i} p_{G i} \cos \theta_{w i} \cos \theta_{G i} \cos \phi_{i}\right]+1
\end{aligned}
$$

and the capacitance of the solution in a parallel plate configuration is obtained to be

$$
C_{\text {sol }}=\frac{\varepsilon_{\text {eff }} \varepsilon_{0} S}{d}
$$

where $d$ is the distance between the plates of the measuring cell and $S$ denotes the area of the electrodes.

The net effective dielectric constant as well as capacitance of the solution is determined by considering the fact that the dipoles are randomly oriented in reference to the applied electric field and also with each other. The theoretical estimation is performed by employing MATLAB, where three sets of random numbers are generated, relevant to angles of the dipoles for water-water, water-glucose and glucose-glucose interactions with reference to the applied electric field. All these angles were chosen randomly for the interval of $-2 \pi$ to $2 \pi$. The dipole moments corresponding to water and glucose molecules were chosen as $1.85 \mathrm{D}$ and 2.89 $\mathrm{D}$, respectively [27-28]. The theoretical values are compared with the experimental data and the results are obtained within $5 \%$ error of the experimental results. 


\section{Experimental}

The capacitance and impedance measurement of the solution with varying glucose concentration was performed using a computer-interfaced LCR meter (TEGAM, Model 3550), where a parallel-plate conductivity cell, with unity cell constant, was connected to the meter as illustrated in Fig. 2(a). The electrical equivalent circuit of the measuring setup is shown in Fig. 2(b). Two capacitors $\left(C_{d l}\right)$ and a resistor $\left(R_{s o l}\right)$ were in series and a third capacitor $\left(C_{s o l}\right)$ was in parallel with the series combination. $C_{d l}$ arises due to the formation of a double-layer on the surface of electrodes and the liquid [29-31]. $C_{\text {sol }}$ and $R_{\text {sol }}$ are the capacitance and resistance, respectively, contributed from the solution due to its dielectric nature. The equivalent impedance of such a circuit is given by,

$$
|Z|=\frac{\left|z_{1}\right| \cdot\left|z_{2}\right|}{\left|z_{1}+z_{2}\right|}
$$

where

$$
z_{1}=R_{s o l}-\frac{j}{\pi f C_{d l}}
$$

and

$$
z_{2}=-\frac{j}{2 \pi f C_{s o l}}
$$

(a)
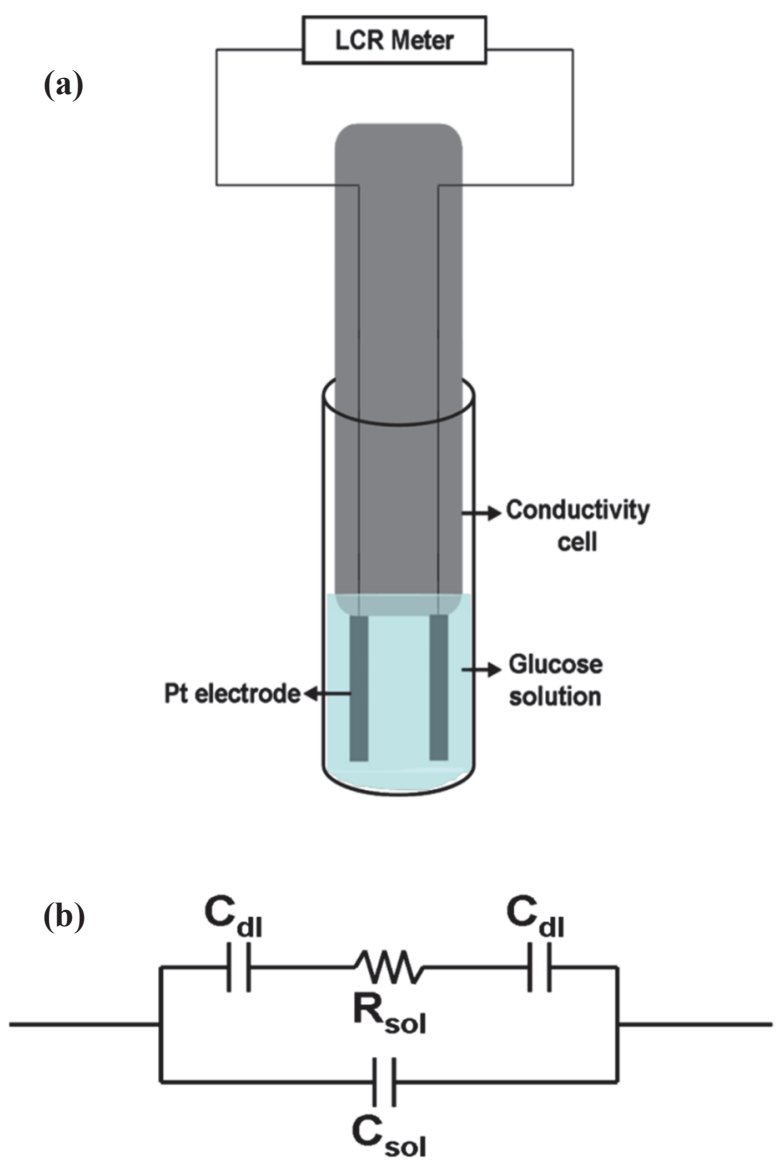

Fig. 2: (a) Schematic diagram of the conductivity cell inside the test tube containing the solution of DI water and glucose; (b) Equivalent circuit of the system.
In such a system, the double layer capacitors dominate the total impedance of the circuit at very low frequencies, resistive part dominates in the mid-frequency range, and the dielectric nature of the solution becomes significant at relatively higher frequency range [31-32]. Electrical measurements were performed at an applied AC bias of $1 \mathrm{~V}$ (peak-to-peak) amplitude in the frequency range of $50 \mathrm{~Hz}$ to $4 \mathrm{MHz}$. The LCR meter was sufficiently equipped with noise reduction techniques during measurements and the impedance and capacitance were measured for thirteen different sets of volume fractions of glucose in DI water.

\section{Results and Discussion}

Fig. 3 (a) - (c) depicts the plots of variation of impedance, capacitance and conductance with volume fraction of glucose in the DI water-glucose solution for the frequencies $1 \mathrm{kHz}, 10 \mathrm{kHz}$ and $100 \mathrm{kHz}$. The experiment has been performed within the volume fraction range of 0.1 to 0.5 . It is apparent from all such plots that the variation of electrical parameters of interest exhibits a quasi-oscillatory behavior with the volume fraction of glucose in the solution, instead of showing a linear variation.

It is worth mentioning that a linear behavior would be observed if the glucose was added into a buffer saline solution [4, 33]. For such verification, a set of experiments was also performed for the glucose volume fraction range of 0.1 to 0.6 with phosphate buffer saline (PBS) as the base solvent. Fig. 4 (a) - (c) depicts the plots of variation of impedance, capacitance and conductance with volume fraction of glucose in the PBS-glucose solution for the frequencies $1 \mathrm{kHz}, 10 \mathrm{kHz}$ and $100 \mathrm{kHz}$, confirming the linear behavior of the electrical parameters with the concentration of glucose.

However, the focus of current work was to develop a comprehensive understanding on the electrical behavior of pure DI water and glucose solution. The quasi-oscillatory behavior of the electrical parameters of the solution, as seen in Fig. 3(a) - (c) is attributed to the random distribution of dipoles present in such a polar solution. Since the dipoles are randomly distributed with respect to the applied electric field, at some composition the projections of the dipoles in the direction of electric field will be more compared to the other compositions, which will lead to higher polarization and hence higher dielectric constant and capacitance.

At $1 \mathrm{kHz}$, the impedance values were measured as 74.4 $\mathrm{k} \Omega, 76 \mathrm{k} \Omega, 52.5 \mathrm{k} \Omega, 80.9 \mathrm{k} \Omega$ and $111 \mathrm{k} \Omega$ for the volume fractions of glucose $0.1,0.2,0.3,0.4$ and 0.5 , respectively. At all the frequencies under test such quasi-oscillatory nature dictates that the capacitance and impedance will have repetitive value for different concentrations of glucose in the solution which may mislead the diagnosis of glucose content in any sample. The theoretical predictions also justify the quasi-oscillatory variation of several electrical parameters such as impedance, capacitance and conductance with glucose concentration in the solution. Since the dielectric behavior of the solution becomes significant at relatively 
high frequencies, the response of these electrical parameters with increasing glucose concentration is presented in the range of $1 \mathrm{kHz}$ to $100 \mathrm{kHz}$.
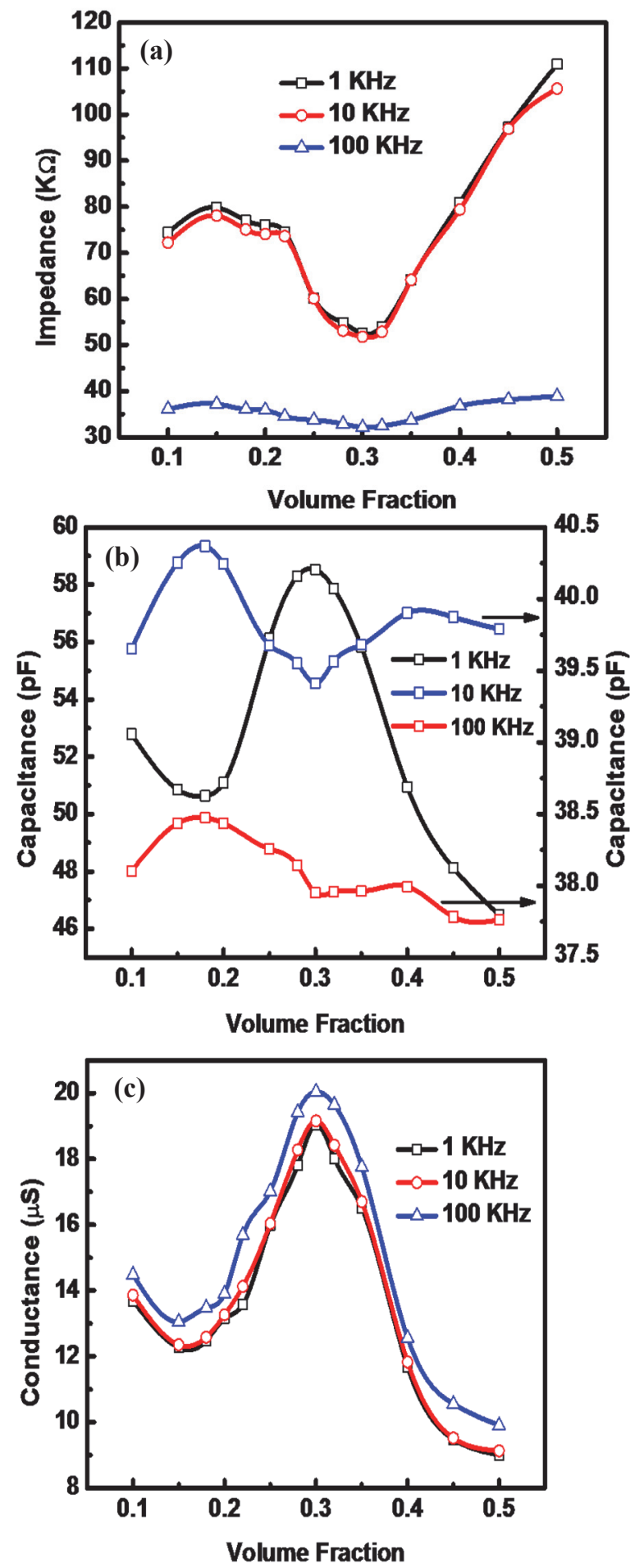

Fig. 3: Plots of the variations of electrical parameters with volume fraction of glucose in DI water: (a) variation of impedance $1 \mathrm{kHz}, 10$ $\mathrm{kHz}$ and $100 \mathrm{kHz}$; (b) variation of capacitance at frequencies $1 \mathrm{kHz}$, $10 \mathrm{kHz}$ and $100 \mathrm{kHz}$ and; (c) variation of conductance at $1 \mathrm{kHz}, 10$ $\mathrm{kHz}$ and $100 \mathrm{kHz}$.
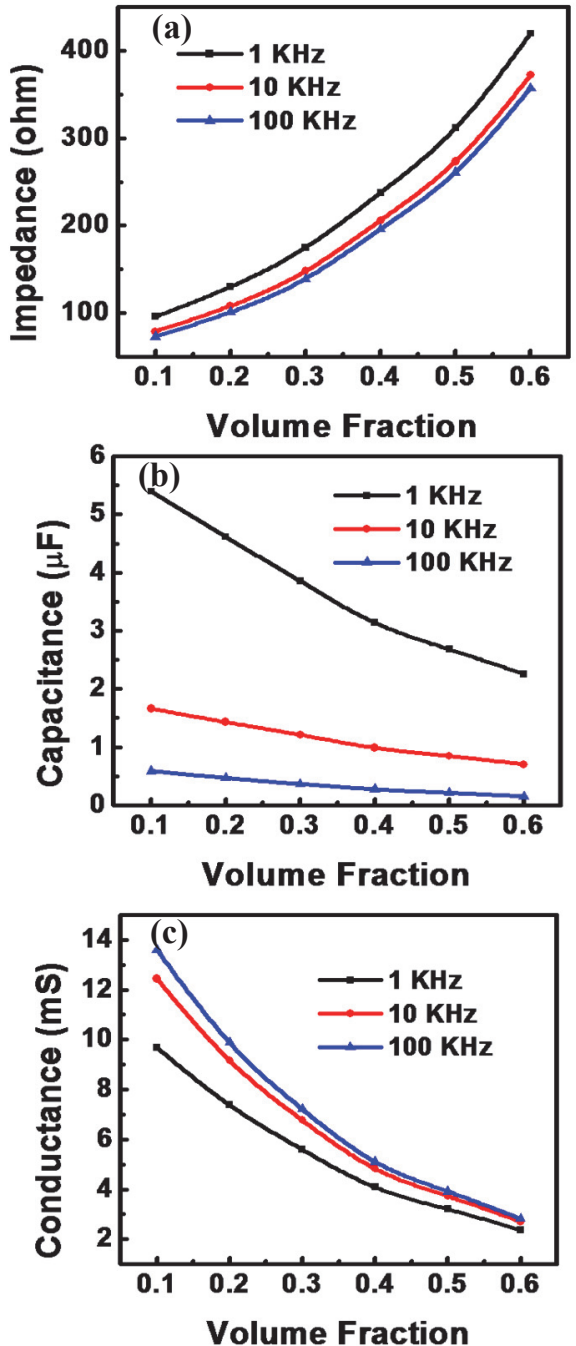

Fig. 4: Plots of the variations of electrical parameters with volume fraction of glucose in PBS: (a) variation of impedance $1 \mathrm{kHz}, 10 \mathrm{kHz}$ and $100 \mathrm{kHz}$; (b) variation of capacitance at frequencies $1 \mathrm{kHz}, 10 \mathrm{kHz}$ and $100 \mathrm{kHz}$ and; (c) variation of conductance at $1 \mathrm{kHz}, 10 \mathrm{kHz}$ and $100 \mathrm{kHz}$.

The experimental results were verified from the theoretical estimation by using the model developed in Eqn. (13) and the comparative plots are shown in Fig. 5(a) and (b), indicating very good agreement between the theoretical and experimental data. Such an agreement suggests that the prediction of randomly distributed molecular dipoles in a DI water-glucose solution is accurate and their randomness is a function of volume fraction of glucose. The higher the volume fraction of glucose the higher will be the waterglucose and glucose-glucose dipoles leading to a change of their previous orientation relevant to a certain volume fraction. This also leads to a change of relative phase angles of the dipoles among themselves and also with the applied electric field. The measurements are performed for ten times by taking fresh DI water and glucose on different occasions and the similar results within a variation of $5 \%$ were obtained. Fresh solutions were used to minimize the effect of hydration shell formation outside the glucose molecules [3436]. 


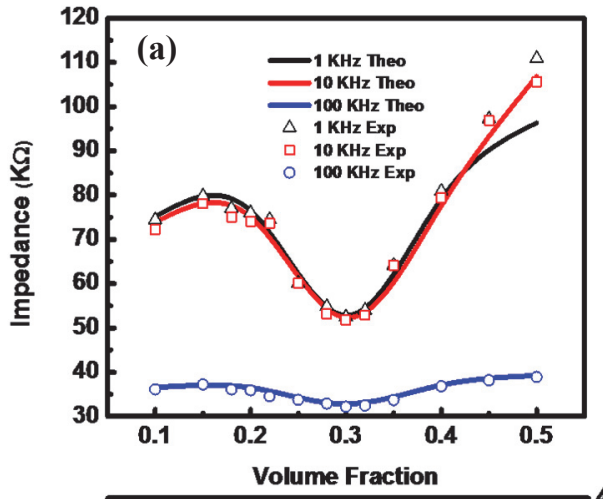

[29-30, 36-37]. However, the capacitance contributed from the bulk solution is observed at higher frequencies, where the influence of Stern layer gets diminished, and such capacitance is measured to be $35.7 \mathrm{pF}-55.2 \mathrm{pF}$ in the higher frequency range, as shown in Fig. 6(b). The conductance spectra of the solutions of different volume fractions of glucose are plotted in Fig. 6(c) for the frequency range considered. The conductance values are measured to be in the range between $8.95 \mu \mathrm{S}$ and $52.9 \mu \mathrm{S}$ for different volume fractions of glucose.
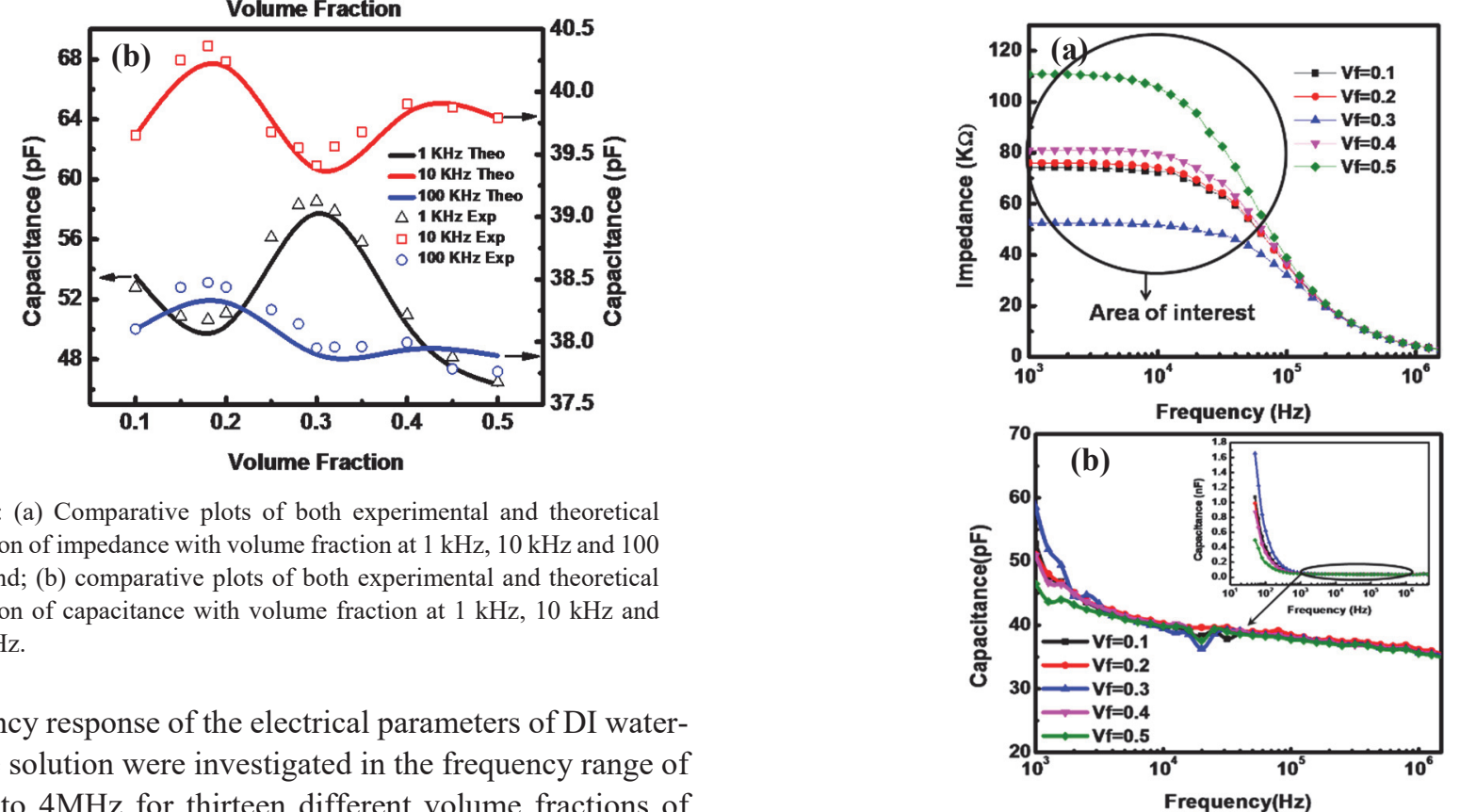

Fig. 5: (a) Comparative plots of both experimental and theoretical variation of impedance with volume fraction at $1 \mathrm{kHz}, 10 \mathrm{kHz}$ and 100 $\mathrm{kHz}$ and; (b) comparative plots of both experimental and theoretical variation of capacitance with volume fraction at $1 \mathrm{kHz}, 10 \mathrm{kHz}$ and $100 \mathrm{kHz}$.

Frequency response of the electrical parameters of DI waterglucose solution were investigated in the frequency range of $100 \mathrm{~Hz}$ to $4 \mathrm{MHz}$ for thirteen different volume fractions of glucose ranging from 0.1 to 0.5 . Fig. 6(a), (b) and (c), depicts the impedance, capacitance, and conductance results for volume fractions $0.1,0.2,0.3,0.4$ and 0.5 , respectively.

The variation of impedance of the solution with frequency is plotted in Fig. 6(a). In the lower frequency range (below $1 \mathrm{kHz}$ ), the value of measured impedance is due to DI water only which is obtained to be $155 \mathrm{k} \Omega$. The measured impedance gets reduced with the addition of glucose and at the medium frequency range, the solution is almost resistive in nature and hence it is frequency independent. At relatively higher frequencies $(>1 \mathrm{kHz})$, the impedance gradually decreases with the increase of measuring frequencies. In this higher frequency range, dielectric nature of the solution dominates the overall impedance of the system. Similar variation of impedance spectrum has been observed for all concentrations of glucose considered and its values have been measured to be within $40 \mathrm{k} \Omega$ to $112 \mathrm{k} \Omega$. The low frequency capacitance of the solution of volume fraction 0.1 is measured to be $1.07 \mathrm{nF}$ and it decreases sharply with increasing frequency. Above the measuring frequency of 1 $\mathrm{kHz}$, the rate of such variation decreases and finally, it becomes almost independent of the measuring frequency above $50 \mathrm{kHz}$. A similar frequency response is observed for the other solutions with different concentrations of glucose. The relatively higher capacitance at low frequencies is attributed to the formation of Stern layer on the electrodes

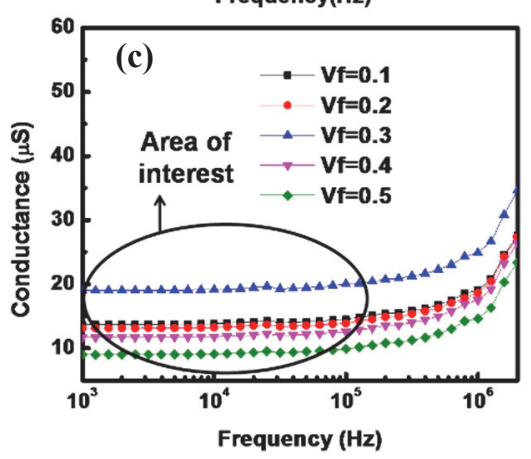

Fig. 6: Plots showing the experimentally measured spectra of impedance, capacitance and conductance for different compositional concentration of glucose in DI water. (a) Impedance over the frequency window emphasizing $1 \mathrm{kHz}$ to $100 \mathrm{kHz}$ range. (b) Capacitance over the entire frequency window (inset) and Capacitance spectrum emphasizing $1 \mathrm{kHz}$ to $1.5 \mathrm{MHz}$ range (c) Conductance over the frequency window emphasizing $1 \mathrm{kHz}$ to $100 \mathrm{kHz}$ range.

\section{Conclusions}

Several electrical parameters such as impedance, capacitance and conductance of DI-water glucose solution with varying glucose content have been measured by employing impedance spectroscopy. The variation of such electrical parameters with the concentration of glucose has been observed to be quasi-oscillatory in nature - the result of 
random variation of alignment of the dipole moments at molecular level for different glucose content in the solution. The quasi-oscillatory nature due to random dipole orientations of water-water, water-glucose and glucoseglucose interaction has also been confirmed by theoretical modeling and the relevant analytical expression has been developed. Therefore, such measurements provide a deterministic approach to identify the overall nature of a glucose containing solution in terms of polarity of the base solvent.

\section{Acknowledgement}

Mr. S. Chakraborty likes to acknowledge the Department of Science and Technology (DST), Government of India for providing the INSPIRE Fellowship to pursue his research. Mr. C. Das and Mr. R. Saha acknowledge the Centre of Excellence (COE), TEQIP, sponsored by World Bank for the funding to support their research. Mr. A. Das acknowledges the UGC fellowship for supporting his research. The authors would also like to acknowledge the funding support from DST PURSE, Government of India for developing the Electrical Characterization Laboratory.

\section{References}

1. Schurr A. Lactate, glucose and energy metabolism in the ischemic brain (Review). International Journal of Molecular Medicine.2002; 10: 131-136.

http://dx.doi.org/10.3892/ijmm.10.2.131

2. Kim NY, Adhikari KK, Dhakal R, Chuluunbaatar Z, Wang C, Kim, E-C. Rapid, Sensitive, and Reusable Detection of Glucose by a Robust Radiofrequency Integrated Device Biosensor Chip. Scientific Reports. 2015; 5: 7807. http://dx.doi.org/10.1038/srep07807

3. Wang J. Electrochemical Glucose Biosensors. Chemical Reviews. 2008; 108: 814-825. http://dx.doi.org/10.1021/cr068123a

4. Yoon G. Dielectric properties of glucose in bulk aqueous solutions: Influence of electrode polarization and modelling. Biosensors and Bioelectronics. 2011; 26: 2347-2353. http://dx.doi.org/10.1016/j.bios.2010.10.009

5. Zhou YG, Yang S, Qian QY, Xia XH. Gold nanoparticles integrated in a nanotube array for electrochemical detection of glucose. Electrochem. Commun. 2009; 11: 216-219. http://dx.doi.org/10.1016/j.elecom.2008.11.010

6. Joshi PP, Merchant SA, Wang YD, Schmidtke DW. Amperometric biosensors based on redox polymer-carbon nanotube-enzyme composites. Anal. Chem. 2005; 77: 31833188. http://dx.doi.org/10.1021/ac0484169

7. Gabriel C. Chapter 3, In: Barnes, F.S., Greenebaum, B. (Eds.), Bioengineering and Biophysical Aspects of Electromagnetic Fields. CRC Press. Boca Raton. 2007; pp. 51-100.

8. Raicu V, Popescu A. Integrated Molecular and Cellular Biophysics. Springer. Dordrecht. 2008. http://dx.doi.org/10.1007/978-1-4020-8268-9
9. Bell GI, Kayano T, Buse JB, Burant CF, Takeda J, Lin D, Fukumoto H, Seino S. Molecular biology of mammalian glucose transporters. Diabetes Care. 1990; 13: 3. http://dx.doi.org/10.2337/diacare.13.3.198

10. Tura A, Sbrignadello S, Barison S, Conti S, Pacini G. Impedance spectroscopy of solutions at physiological glucose concentrations. Biophysical Chemistry. 2007; 129: 235-241. http://dx.doi.org/10.1016/j.bpc.2007.06.001

11. Tura A. Reply to comment on experimental methods and conclusions of impedance spectroscopy of solutions at physiological glucose concentrations by A. Caduff, M. S. Talary, Y. Feldman. Biophysical Chemistry. 2008; 132: 167168. http://dx.doi.org/10.1016/j.bpc.2007.10.006

12. Pradhan R, Mitra A, Das S. Quantitative evaluation of blood glucose concentration using impedance sensing devices. Journal of Electrical Bioimpedance. 2013; 4: 73-77. http://dx.doi.org/10.5617/jeb.657

13. Tura A, Sbrignadello S, Cianciavicchia D, Pacini G, Ravazzani P. A Low Frequency Electromagnetic Sensor for Indirect Measurement of Glucose Concentration: In Vitro Experiments in Different Conductive Solutions. Sensors. 2010; 10: 5346-5358. http://dx.doi.org/10.3390/s100605346

14. Felderhof BU, Ford GW, Cohen EGD. The Clausius-Mossotti Formula and Its Nonlocal Generalization for a Dielectric Suspension of Spherical Inclusions. Journal of Statistical Physics. 1983; 33: 241-260. http://dx.doi.org/10.1007\%2FBF01009796

15. Debye P. Polar molecules. New York, The Chemical Catalogue Company, Dover. 1929.

16. Onsager L. Electric Moments of Molecules in Liquids. Journal of American Chemical Society. 1936; 58: 1486-1493. http://dx.doi.org/10.1021/ja01299a050

17. Kirkwood JG. The dielectric polarization of polar liquids. Journal of Chemical physics. 1939; 7: 911. http://dx.doi.org/10.1063/1.1750343

18. Huang K, Yang X. A method for calculating the effective permittivity of a mixture solution during a chemical reaction by experimental results. Progress in Electromagnetics Research Letters. 2008; 5: 99-107. http://dx.doi.org/10.2528/PIERL08110403

19. Höchtl P, Boresch S, Steinhausera O. Dielectric properties of glucose and maltose solutions. Journal of Chemical Physics. 2000; 112: 9810. http://dx.doi.org/10.1063/1.481619

20. Mazurkiewicz J, Tomasik P. Effect of external electric field upon charge distribution, energy and dipole moment of selected monosaccharide molecules. Natural Science. 2012; 4: 276-285. http://dx.doi.org/10.4236/ns.2012.45040

21. Liao X, Raghavana GSV, Dai J, Yaylayan VA. Dielectric properties of $\alpha$-d-glucose aqueous solutions at $2450 \mathrm{MHz}$, Food Research International. 2003; 36: 485-490. http://dx.doi.org/10.1016/S0963-9969(02)00196-5

22. Liao X, Raghavana VGS, Meda V, Yaylayan VA. Dielectric properties of supersaturated $\alpha$-D-glucose aqueous solutions at $2450 \mathrm{MHz}$. Journal of Microwave Power \& Electromagnetic Energy. 2001; 36: 131-138. 
23. Griffiths DJ. Introduction to Electrodynamics, forth ed. Prentice-Hall, Inc., New Jersey. 2012.

24. Jackson JD. Classical Electrodynamics, third ed. John Wiley \& Sons, Inc. 1998.

25. Boresch S, Ringhofer S, Höchtl P, Steinhausera O. Towards a better description and understanding of biomolecular salvation. Biophysical Chemistry. 1999; 78: 43-68. http://dx.doi.org/10.1016/S0301-4622(98)00235-X

26. Steiner T. The Hydrogen Bond in the Solid State. Angew. Chem. Int. Ed. 2002; 41: 48-76. http://dx.doi.org/10.1002/15213773(20020104)41:1<48::AID-ANIE48>3.0.CO;2-U

27. Jatkar SKK, Iyenger BRY. Dielectric Constants and Molecular Structure. J. Indian Inst. Sci. 1946; 28A. Part II: p. 1.

28. Ramakrishna V. Dipole Moments of Two Derivatives of DGlucose in Liquid State: Application of Jatkar's Equation. Journal of the Indian Institute of Science. 1953; 35: 4.

29. Bonthuis DJ, Gekle S, Netz RR. Dielectric Profile of Interfacial Water and its Effect on Double-Layer Capacitance. Physical Review Letter. 2011; 107: 166102. http://dx.doi.org/10.1103/PhysRevLett.107.166102

30. Barbieri O, Hahn M, Herzog A, Kötz R. Capacitance limits of high surface area activated carbons for double layer capacitors. Carbon. 2005; 4: 1303-1310. http://dx.doi.org/10.1016/j.carbon.2005.01.001

31. Zou Z, Kai J, Rust MJ, Han J, Ahn CH. Functionalized nano interdigitated electrodes arrays on polymer with integrated microfluidics for direct bio-affinity sensing using impedance measurement. Sensors and Actuators A. 2007; 136: 518-526. http://dx.doi.org/10.1016/j.sna.2006.12.006

32. Ibrahim M, Claudel J, Kourtiche D, Nadi M. Geometric parameters optimization of planar interdigitated electrodes for bioimpedance spectroscopy. Journal of Electrical Bioimpedance. 2013; 4: 13-22. http://dx.doi.org/10.5617/jeb.304

33. Shervedani RK, Mehrjardi AH, Zamiri N. A novel method for glucose determination based on electrochemical impedance spectroscopy using glucose oxidase self-assembled biosensor. Bioelectrochemistry. 2006; 69: 201-208. http://dx.doi.org/10.1016/j.bioelechem.2006.01.003

34. Suzuki T. The hydration of glucose: the local configurations in sugar-water hydrogen bonds. Phys. Chem. Chem. Phys. 2008; 10: 96-105. http://dx.doi.org/10.1039/B708719E

35. Franks F. Physical chemistry of small carbohydrates equilibrium solution properties. Pure Appl. Chem. 1987; 59: 1189-1202. http://dx.doi.org/10.1351/pac198759091189

36. Carrique F, Arroyo FJ, Delgado AV. Electrokinetics of Concentrated Suspensions of Spherical Colloidal Particles: Effect of a Dynamic Stern Layer on Electrophoresis and DC Conductivity. Journal of Colloid and Interface Science. 2001; 243: 351-361. http://dx.doi.org/10.1006/jcis.2001.7903

37. Wang H, Pilon L. Accurate Simulations of Electric Double Layer Capacitance of Ultramicroelectrodes. J. Phys. Chem. 2011; 115: 16711-16719. http://dx.doi.org/10.1021/jp204498e 